Canadian Journal of Physics

Revue canadienne de physique

\title{
Entropic uncertainty relation under correlated dephasing channels
}

\begin{tabular}{|r|l|}
\hline Journal: & Canadian Journal of Physics \\
\hline Manuscript ID & cjp-2017-0683.R1 \\
\hline Manuscript Type: & Article \\
\hline Date Submitted by the Author: & 23-Nov-2017 \\
\hline Complete List of Authors: & Karpat, Göktuğ; Izmir Ekonomi Universitesi, \\
\hline $\begin{array}{r}\text { Is the invited manuscript for } \\
\text { consideration in a Special } \\
\text { Issue? : }\end{array}$ & $\begin{array}{l}\text { Entropic Uncertainty Relations, Open Quantum Systems, Dephasing } \\
\text { Channels, Correlated Channels, Channels with Memory }\end{array}$ \\
\hline \multicolumn{2}{|r}{} \\
\hline
\end{tabular}

\section{SCHOLARONE ${ }^{\text {TW }}$}

Manuscripts 


\title{
Entropic uncertainty relation under correlated dephasing channels
}

\author{
Göktuğ Karpat* \\ Faculty of Arts and Sciences, Department of Physics, Izmir University of Economics, İmir, 35330, Turkey
}

\begin{abstract}
Uncertainty relations are one of the characteristic traits of quantum mechanics. Even though the traditional uncertainty relations are expressed in terms of the standard deviation of two observables, there exists another class of such relations based on entropic measures. Here we investigate the memory-assisted entropic uncertainty relation in an open quantum system scenario. We study the dynamics of the entropic uncertainty and its lower bound, related to two incompatible observables, when the system is affected by noise which can be described by a correlated Pauli channel. In particular, we demonstrate how the entropic uncertainty for these two incompatible observables can be reduced as the correlations in the quantum channel grow stronger.
\end{abstract}

PACS numbers: 03.65.Yz, 03.65.Ta, 03.67.Mn

\section{INTRODUCTION}

Quantum systems are typically not isolated from their surrounding environment in real world conditions. The unpreventable interaction between the principal quantum system of interest and its environment is detrimental to the characteristic quantum traits, such as coherence and entanglement in the principal system. The theory of open quantum systems supplies the mathematical tools to comprehend and rigorously analyse such systems, which are undergoing decoherence due to the uncontrollable coupling with the environment [1]. Open quantum system dynamics of various quantum properties have been widely investigated in the recent literature, e.g., quantum entanglement [2] and more general discord-like quantum correlations [3] in composite systems, and quantum coherence [4]. In addition, numerous techniques have been developed to suppress the destructive effects of decoherence on these exclusively quantum mechanical properties, since they are known to serve as a critical resource for quantum computation and quantum information processing tasks.

On the other hand, a very fundamental property of quantum theory is the fact that it is impossible to prepare a quantum state, for which the measurement outcomes of two incompatible observables are arbitrarily accurate. After the pioneering ideas of Heisenberg on uncertainty [5], Kennard rigorously proved the first preparation uncertainty relation for the position $x$ and momentum $p_{x}$ of a particle [6], in terms of the product of standard deviations of these two observables, as $\Delta x \Delta p_{x} \geq \hbar / 2$. Soon after, Robertson generalized it to the case of two arbitrary observables $Y$ and $Z$ showing that [7]

$$
\Delta Y \Delta Z \geq \frac{1}{2}|\langle\psi|[Y, Z]| \psi\rangle| .
$$

Although these type of standard deviation based uncertainty relations have been of great importance for the development of quantum theory, they suffer from several issues limiting their applicability [8]. In particular, the lower bound of uncertainty given in Eq. (1) is state dependent, and for the quantum states $|\psi\rangle$ having a zero expectation value for the commutator $[Y, Z]$, the lower bound vanishes and becomes completely trivial.

\footnotetext{
*goktug.karpat@ieu.edu.tr
}

A possible remedy for these issues is to utilize entropic measures to quantify the amount of uncertainty related to the lack of knowledge of possible measurement results. Despite the first entropic uncertainty relation was introduced by Deutsch [9], one of the most well known version in the literature was proven by Maasen and Uffink [10], which reads

$$
H(Q)+H(R) \geq \log _{2} \frac{1}{c}
$$

The Shannon entropy $H(X)=-\sum_{x} p(x) \log _{2} p(x)$ quantifies the uncertainty related to the observable $X \in(Q, R)$ before its measurement outcome is known, and $p(x)$ is the probability of obtaining the outcome $x$ for a density operator $\rho$ measured in the $X$-basis. $1 / c=1 / \max _{i, j}\left|\left\langle\psi_{i} \mid \phi_{j}\right\rangle\right|^{2}$ denotes the complementarity of the observables $Q$ and $R$, where $\left|\psi_{i}\right\rangle$ and $\left|\phi_{j}\right\rangle$ are respectively the eigenstates of $Q$ and $R$.

In this work, we intend to investigate the open quantum system dynamics of the memory-assisted entropic uncertainty relation. In fact, this problem has been recently studied for several different decoherence models exhibiting pure dephasing and relaxation type dynamics [11-13]. However, possible effects of classically formed correlations in the application of quantum channels, which describes the decoherence process, have not yet been considered. Here, we will systematically explore the consequences of having correlated noise on the considered quantum system for the behaviour of the entropic uncertainty of two incompatible observables. Specifically, we will demonstrate that both the entropic uncertainty and its lower bound can be reduced as we induce higher degree of correlations in the quantum noise channel.

\section{MEMORY-ASSISTED UNCERTAINTY RELATION}

Let us first mention that it is actually informative and convenient to think about the entropic uncertainty relation given in Eq. (2) in terms of an uncertainty game played between two players, namely, Alice and Bob. Before the game commences, Alice and Bob agree to only use two fixed observables $Q$ and $R$. Bob then prepares a particle in a state of his choosing and sends it to Alice, who in return measures the particle she received in one of the two fixed bases and tells her choice to Bob. The goal of the game for Bob is to minimize 
the amount of uncertainty related to the outcomes of Alice's measurement. Indeed, as Bob is only able to access classical information in this scenario, Eq. (2) puts a bound Bob's uncertainty about the measurement outcomes that Alice obtains.

We now consider a generalization of the above setting by assuming that Bob gains access to an additional particle $B$ serving as a quantum memory, which is entangled with the particle $A$ that Alice receives. It has been recently demonstrated that, with the introduction of the quantum memory particle $B$, it is possible to formulate the following memoryassisted entropic uncertainty relation [14]:

$$
S(Q \mid B)+S(R \mid B) \geq \log _{2} \frac{1}{c}+S(A \mid B),
$$

where $S(A \mid B)=S\left(\rho_{A B}\right)-S\left(\rho_{B}\right)$ is the conditional entropy. Whereas $S(\rho)=-\operatorname{tr}\left[\rho \log _{2} \rho\right]$ is the von Neumann entropy, $S(X \mid B)$ with $X \in(Q, R)$ are the conditional entropies of the post-measurement states $\rho_{X B}=\sum_{j}\left(\left|\psi_{j}\right\rangle\left\langle\psi_{j}\right| \otimes\right.$ I) $\rho_{A B}\left(\left|\psi_{j}\right\rangle\left\langle\psi_{j}\right| \otimes \mathbb{I}\right)$ after the subsystem $A$ is measured in $X$ basis. $\left\{\left|\psi_{j}\right\rangle\right\}$ are the eigenstates of the observable $X$, and $\mathbb{I}$ represents the identity matrix. In this new setting, the memory-assisted entropic uncertainty relation given in Eq. (3) becomes what restricts the uncertainty of Bob about Alice's measurement outcomes in $Q$ and $R$ bases. While the on the left-hand side of Eq. (3) we have Bob's total uncertainty about Alice's measurement results, on the right-hand side we have complementarity and the quantum conditional entropy $S(A \mid B)$, which might potentially reduce the lower bound of Bob's uncertainty related to $Q$ and $R$, since quantum conditional entropy can take negative values unlike its classical analogue. In fact, provided that Alice and Bob share a maximally entangled state, then Bob can predict the measurement outcomes of two incompatible observables with zero uncertainty. It is also worth to mention that the relation of quantum and classical correlations, and the memory-assisted entropic uncertainty relation has been investigated in the literature from different perspectives $[15,16]$. In particular, an improved and a potentially tighter bound has also been obtained [17].

\section{CORRELATED PAULI CHANNELS}

In this section, we introduce the correlated channels (also known as channels with memory in the literature) which will describe the open system dynamics of our principal quantum system of interest. A Pauli channel $\mathcal{E}$ acting on a single qubit is simply a random application of Pauli transformations,

$$
\rho \rightarrow \mathcal{E}(\rho)=\sum_{i=0}^{3} q_{i} \sigma_{i} \rho \sigma_{i},
$$

where $\sigma_{i}$ 's are the Pauli operators in $x, y, z$ directions and the $\sigma_{0}$ is the $2 \times 2$ identity matrix. $\rho$ denotes the density matrix of the open system and $q_{i}$ 's form a probability distribution as $\sum_{i=0}^{3} q_{i}=1$. Throughout this work, we consider only two consequent implementations of the channel for simplicity.
Then, the Pauli channel acts on a two-qubit system as follows

$$
\rho \rightarrow \mathcal{E}(\rho)=\sum_{i, j=0}^{3} p_{i j}\left(\sigma_{i} \otimes \sigma_{j}\right) \rho\left(\sigma_{i} \otimes \sigma_{j}\right)
$$

where $p_{i j}$ is a joint probability distribution. In case we assume that $p_{i j}$ can be factorized as $p_{i j}=q_{i} q_{j}$, the noise is not correlated and Eq. (5) in fact describes two independent applications of the channel on the two-qubit state. On the other hand, $p_{i j}$ is indeed not restricted to be factorized and such a situation might give rise to correlations between the two repeated applications of the channel.

One of the well known models, which takes the correlations in the application of the Pauli channels into the account, has been introduced by Macchiavello and Palma [18], where the joint probability distribution is given by

$$
p_{i j}=(1-\mu) q_{i} q_{j}+\mu q_{i} \delta_{i j} .
$$

Here the parameter $\mu \in[0,1]$ quantifies the amount of classical correlations in the implementation of the channel. Particularly, existence of a non-vanishing $\mu$ actually forces the same Pauli operator to be re-applied in the second use of the channel with some probability. The quantum channel is maximally correlated in case we have $\mu=1\left(p_{i j}=q_{i} \delta_{i j}\right)$ since it is guaranteed that the same Pauli transformation is applied on both qubits. On the other hand, it is straightforward to see that we recover the case of two independent channels for $\mu=0$.

Until this point, we have elaborated on the structure of the rather general case of correlated Pauli channels. Let us now introduce a specific physical model which describes coloured pure dephasing dynamics of a single qubit [19], and which has a solution in the form of Eq. (4). Suppose that the time evolution of the qubit can be described with a time-dependent Hamiltonian $H(t)=\hbar \Gamma(t) \sigma_{z}$, and $\Gamma(t)$ is an independent random variable having the statistics of a random telegraph signal. If we assume that $\Gamma(t)=\alpha n(t)$, where $n(t)$ has a Poisson distribution with the mean $\nu=t / 2 \tau$ and $\alpha$ is a coinflip random variable having the possible values \pm 1 , then the time evolution of the open system composing of a single qubit can be obtained using the operator-sum representation

$$
\rho(\nu)=\sum_{i=1}^{2} K_{i}(\nu) \rho(0) K_{i}(\nu)^{\dagger},
$$

where the corresponding Kraus operators are given as

$$
\begin{aligned}
& K_{1}(\nu)=\sqrt{[1+\Phi(\nu)] / 2} \mathbb{I}, \\
& K_{2}(\nu)=\sqrt{[1-\Phi(\nu)] / 2} \sigma_{3},
\end{aligned}
$$

with $\Phi(\nu)=e^{-\nu}[\cos (u \nu)+\sin (u \nu) / u], u=\sqrt{(4 \tau)^{2}-1}$, and $\nu=t / 2 \tau$ is the scaled time.

We can now easily see that the solution of this coloured pure dephasing model is in the form of Eq. (4) for a single qubit, where the time dependent coefficients $q_{i}$ 's can be identified as

$$
q_{0}=\frac{1}{2}[1+\Phi(\nu)], \quad q_{1}=q_{2}=0, \quad q_{3}=\frac{1}{2}[1-\Phi(\nu)] .
$$



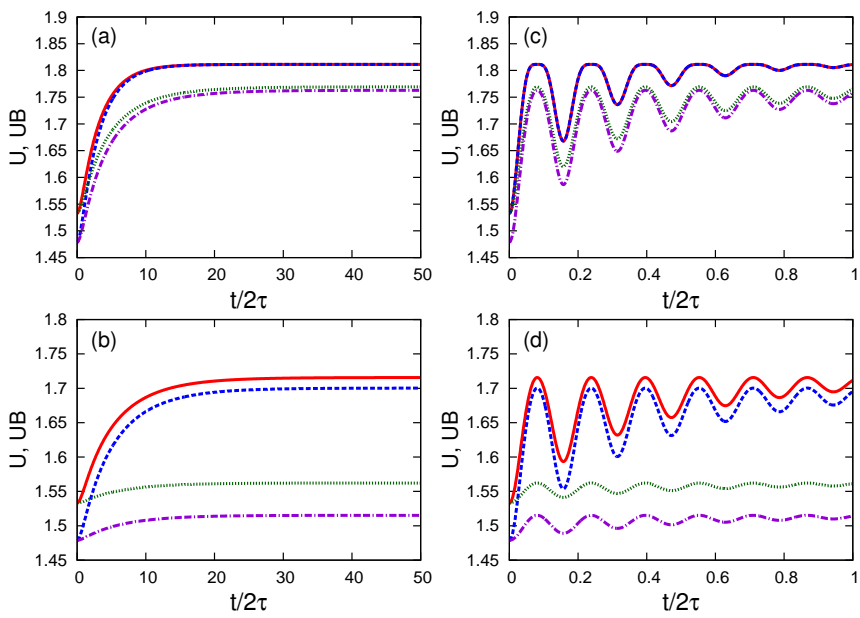

FIG. 1. The incompatible observables are chosen as $Q=\sigma_{1}$ and $R=\sigma_{3}$ and the initial Bell-diagonal state has the parameters $c_{1}=-0.6, c_{2}=c_{3}=0.5$. The model parameter controlling nonMarkovianity is assumed to be $\tau=0.1$ in (a) and (b), and $\tau=5$ in (c) and (d). In (a) and (c), we show the dynamics of the uncertainty $U$ for $\mu=0$ (red solid line) and for $\mu=0.4$ (dotted green line), and the lower bounds $U B$ for $\mu=0$ (blue dashed line) and for $\mu=0.4$ (purple dot-dashed line). In (b) and (d), we show the dynamics of the uncertainty $U$ for $\mu=0.6$ (red solid line) and for $\mu=0.95$ (dotted green line), and their lower bounds $U B$ for $\mu=0.6$ (blue dashed line) and for $\mu=0.95$ (purple dot-dashed line).

Thus, the dephasing type correlated Pauli channel acting on a two-qubit quantum system (which is in the form of Eq. (5)) can be simply described in the following way:

$$
\begin{aligned}
\mathcal{E}(\rho) & =p_{03}\left(\sigma_{0} \otimes \sigma_{3}\right) \rho\left(\sigma_{0} \otimes \sigma_{3}\right)+p_{30}\left(\sigma_{3} \otimes \sigma_{0}\right) \rho\left(\sigma_{3} \otimes \sigma_{0}\right) \\
& +p_{00}\left(\sigma_{0} \otimes \sigma_{0}\right) \rho\left(\sigma_{0} \otimes \sigma_{0}\right)+p_{33}\left(\sigma_{3} \otimes \sigma_{3}\right) \rho\left(\sigma_{3} \otimes \sigma_{3}\right),
\end{aligned}
$$

Adjusting the parameter $\mu$ will let us vary the degree of correlations between two applications of this dephasing channel.

\section{MAIN RESULTS}

Having briefly discussed the type of classically correlated quantum noise channel we are interested in, we can now analyse the memory-assisted entropic uncertainty relation in this setting. Let us start by introducing the initial bipartite states that we consider in this work, i.e. the Bell-diagonal states,

$$
\rho_{A B}=\frac{1}{4}\left(\mathbb{I}_{4}+\sum_{j=1}^{3} c_{j} \sigma_{j}^{A} \otimes \sigma_{j}^{B}\right),
$$

where $\mathbb{I}_{4}$ denotes the $4 \times 4$ identity matrix, $c_{j}$ are real numbers satisfying $0 \leq\left|c_{j}\right| \leq 1$. Here, the necessarily non-negative eigenvalues of $\rho_{A B}$ can be easily found as

$$
\begin{aligned}
\lambda_{1,2} & =\frac{1}{4}\left(1 \pm c_{1} \pm c_{2}-c_{3}\right), \\
\lambda_{3,4} & =\frac{1}{4}\left(1 \pm c_{1} \mp c_{2}+c_{3}\right) .
\end{aligned}
$$
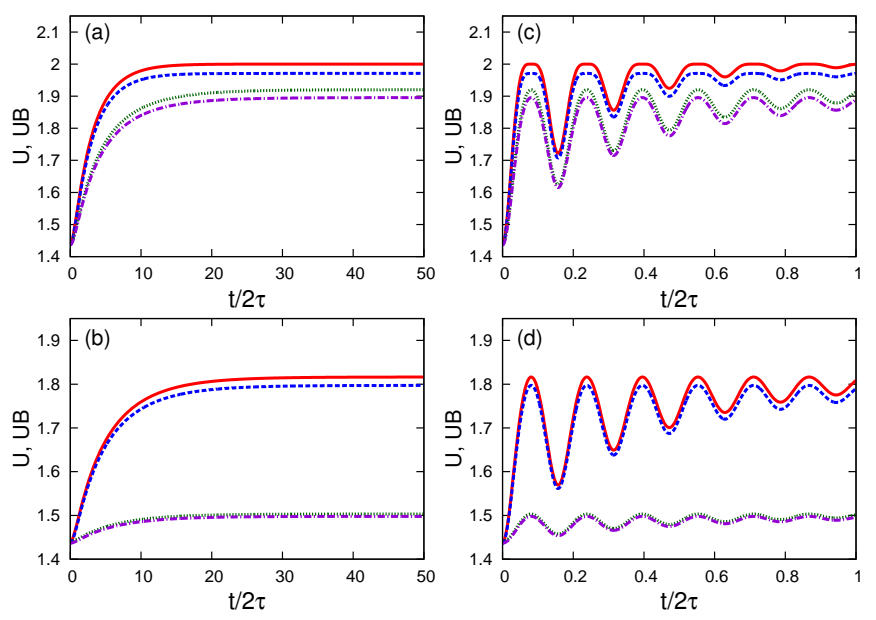

FIG. 2. The incompatible observables are chosen as $Q=\sigma_{1}$ and $R=\sigma_{2}$ and the initial Bell-diagonal state has the parameters $c_{1}=$ $-c_{3}=-0.2, c_{2}=0.8$. The model parameter controlling nonMarkovianity is assumed to be $\tau=0.1$ in (a) and (b), and $\tau=5$ in (c) and (d). In (a) and (c), we show the dynamics of the uncertainty $U$ for $\mu=0$ (red solid line) and for $\mu=0.4$ (dotted green line), and the lower bounds $U B$ for $\mu=0$ (blue dashed line) and for $\mu=0.4$ (purple dot-dashed line). In (b) and (d), we show the dynamics of the uncertainty $U$ for $\mu=0.6$ (red solid line) and for $\mu=0.95$ (dotted green line), and their lower bounds $U B$ for $\mu=0.6$ (blue dashed line) and for $\mu=0.95$ (purple dot-dashed line).

Moreover, it is not difficult to verify that the density matrix describing the dynamics of the correlated dephasing noise channel given in Eq. (11) can be expressed as

$$
\rho_{A B}(t)=\rho_{A B} \circ\left(\begin{array}{cccc}
1 & \Phi(\nu) & \Phi(\nu) & \Gamma(\nu, \mu) \\
\Phi(\nu) & 1 & \Gamma(\nu, \mu) & \Phi(\nu) \\
\Phi(\nu) & \Gamma(\nu, \mu) & 1 & \Phi(\nu) \\
\Gamma(\nu, \mu) & \Phi(\nu) & \Phi(\nu) & 1
\end{array}\right)
$$

where $\Gamma(\nu, \mu)=\mu-\Phi(\nu)^{2}(\mu-1)$. At this point, it is worth to mention that the above described time-evolution preserves the general form of Bell-diagonal states and transforms their three real coefficients as follows:

$$
c_{1}(t)=c_{1} \Gamma(\nu, \mu), \quad c_{2}(t)=c_{2} \Gamma(\nu, \mu), \quad c_{3}(t)=c_{3} .
$$

Besides, we will assume that the two observables $Q$ and $R$ to be measured by Alice are chosen as pairs of Pauli matrices $\left\{\sigma_{i}\right\}$ with $i \in[1,2,3]$. In this case, the conditional von Neumann entropy $S\left(\sigma_{i} \mid B\right)$, after the particle $A$ is measured by Alice, can be written with the help of the binary entropy function as $S(Q \mid B)=H_{b i n}\left[\left(1+c_{i}\right) / 2\right]$ [11], where $H_{b i n}(x)=-x \log _{2}(x)-(1-x) \log _{2}(1-x)$. Thus, for two different Pauli observables $\sigma_{i}$ and $\sigma_{j}$, the left-hand side of the Eq. (3) or equivalently the total uncertainty is given by $U=H_{b i n}\left[\left(1+c_{i}\right) / 2\right]+H_{b i n}\left[\left(1+c_{j}\right) / 2\right]$. Since we know how the coefficients are transformed by the effect of the correlated dephasing noise from Eq. (15), we can calculate the total uncertainty $U$ straightforwardly. On the other hand, complementarity of $Q$ and $R$ simply becomes $c=1 / 2$ as pairs of Pauli observables are complementary. In turn, the entropic 
uncertainty bound $U B=\log _{2}[1 / c]+S(A \mid B)$ reduces to von Neumann entropy $S\left(\rho_{A B}\right)=-\sum_{i=1}^{4} \lambda_{i} \log \lambda_{i}$ due to the fact that all the Bell-diagonal states have maximally mixed reduced density matrices, that is, $S\left(\rho_{B}\right)=1$. Once again, with the assistance of Eq. (15), it is not difficult to evaluate the dynamics of the lower bound of the uncertainty $S\left(\rho_{A B}(t)\right)$ as we can find out how the eigenvalues given in Eq. (13) are transformed by the action of the correlated noise.

In the following, we will demonstrate how it is possible to reduce the total entropic uncertainty of the incompatible observables $Q$ and $R$ in an open quantum systems scenario via the classical correlations in the application of purely dephasing noise. Let us first recall that the coloured dephasing model we consider here might exhibit memory effects depending on the value of the parameter $\tau$. In case $\tau<1 / 4$, the dynamics is Markovian and there exists no memory effects. However, when we have $\tau>1 / 4$, memory effects emerge due to the non-Markovian nature of the dynamics [20].

In Fig. 1, we display the time evolution of the total entropic uncertainty $U$ (along with its lower bound $U B$ ) of Bob about the measurement outcomes of Alice under a correlated dephasing noise setting. In other words, we assume that both the particle $A$, which is to be sent to Alice by Bob, and the memory particle $B$ that Bob can access, are open quantum systems being affected by a correlated dephasing noise. In fact, Fig. 1 shows entropic uncertainty $U$ and its lower bound $U B$ for four different values of the correlation parameter $\mu$ and two different values of the model parameter $\tau$, controlling the Markovianity of the dynamics. We suppose that the initial Bell-diagonal state has the coefficients $c_{1}=-0.6$, $c_{2}=c_{3}=0.5$, and the two observables are fixed as $Q=\sigma_{1}$ and $R=\sigma_{3}$. In Fig. 1(a) and 1(b), dynamical behaviour of $U$ and $U B$ are shown for the Markovian case, where we have no backflow of information from the environment. In particular, while we have the results of our investigation for $\mu=0$ and $\mu=0.4$ in Fig. 1(a), the outcomes of the same analysis for $\mu=0.6$ and $\mu=0.95$ are shown in Fig. 1(b). Here, we can make the observation that, as the parameter $\mu$ (which adjusts the strength of the correlation between the two applications of the channel) increases, both the lower bound $U B$ and the total entropic uncertainty $U$ are diminished. It means that the existence of classical correlations in the two-qubit channel causes a reduction of the total uncertainty, as compared to the case of independent channels where $\mu=0$. Also, it is interesting to see that the lower bound is becoming less tight with the increasing correlations in the channel. In Fig. 1(c) and 1(d), we demonstrate the results of the same analysis for nonMarkovian dynamics as $\tau=5$ and we can clearly observe the revivals in entropic quantities due to the memory effects. It is rather straightforward to observe that similar conclusions hold for this case, that is, the greater the correlations in the dephasing channel is, the less the total uncertainty of the observables will be. Thus, classical correlations in the channel as described in Eq. (6) can reduce the total uncertainty that Bob has about the measurement outcomes of Alice both in Markovian and non-Markovian dephasing noise settings. The underlying physical reason, which makes this reduction possible, has its roots in the residual coherence contained in the composite state $\rho_{A B}$ shared by Alice and Bob as the correlated dephasing noise affects the system. Considering the fact that here we deal with X-shaped states in the Bell-diagonal form and looking at Eq. (14), one can see that all the offdiagonal coherence terms are multiplied by the decay factor $\Gamma(\nu, \mu)=\mu-\Phi(\nu)^{2}(\mu-1)$ and the diagonal population terms remain invariant due to the dephasing interaction. Then, it is not difficult to notice that a greater amount of channel correlations, quantified via the parameter $\mu$, gives rise to a greater amount of coherence left in the state, which in turn makes possible to reduce the entropic uncertainty and its lower bound as their time evolutions are determined by the dynamics of the coherence terms of the density matrix.

Fig. 2 describes the results of the same examination for a different pair of observables and a different initial state, that is, here it is assumed that we have an initial Bell-diagonal state with the coefficients $c_{1}=-c_{3}=-0.2, c_{2}=0.8$, and the two observables are chosen as $Q=\sigma_{1}$ and $R=\sigma_{2}$. Once again, the dynamical behaviour of the total uncertainty $U$ and its lower bound $U B$ are shown for the Markovian case $(\tau=0.1)$ in Fig. 2(a) and Fig. 2(b) for the correlation parameters $\mu=0,0.4$ and $\mu=0.6,0.95$, respectively. When it comes to the non-Markovian case where $\tau=5$, Fig. 2(c) and Fig. 2(d) respectively present the results for $\mu=0,0.4$ and $\mu=0.6,0.95$. Observing the figure, one can conclude that both in Markovian and non-Markovian correlated dephasing noise cases, the total entropic uncertainty $U$ decreases as the strength of the correlations in the implementation of the channel is increased. It is rather interesting to note that whereas the channel correlations have a greater impact in reducing the entropic uncertainty and its lower bound in Fig. 2 as compared to Fig. 1, the uncertainty lower bound gets more and more tight with increasing correlations in Fig. 2, unlike the case of Fig. 1, where increasing channel correlations makes the lower bound less tight. All the same, in both cases, the total amount of uncertainty of Bob about the possible outcomes of the measurements of Alice on the particle $A$ is reduced, when the purely dephasing channel becomes more correlated.

\section{CONCLUSION}

In summary, we studied the open system dynamics of the memory-assisted entropic uncertainty relation when the particle $A$ and the memory $B$ are affected by a classically correlated dephasing channel. We have considered both the Markovian and the non-Markovian time evolutions and two different pairs of observables and in all cases we concluded that as the correlations in the application of the channels is strengthened, the total uncertainty of Bob about the measurement outcomes of Alice is diminished. Lastly, we should mention as a side note that our preliminary analysis shows that the improved uncertainty bound in Ref. [17] behaves similarly to the standard one, i.e., diminishes with increasing channel correlations. Considering that the memory-assisted uncertainty relation has potential to give rise to applications related to witnessing entanglement and cryptographic security [14], we believe that our results might be of relevance in appropriate settings. 
[1] H.-P. Breuer and F. Petruccione, The Theory of Open Quantum Systems, (Oxford Univ. Press, 2007).

[2] R. Horodecki, P. Horodecki, M. Horodecki, and K. Horodecki, Rev. Mod. Phys. 81, 865 (2009).

[3] K. Modi, A. Brodutch, H. Cable, T. Paterek, and V. Vedral, Rev. Mod. Phys. 84, 1655 (2012).

[4] A. Streltsov, G. Adesso, M. B. Plenio, arXiv:1609.02439v3.

[5] W. Heisenberg, Z. Phys. 43, 173 (1927).

[6] E. H. Kennard, Z. Phys. 44, 326 (1927).

[7] H. P. Robertson Phys. Rev. 34, 163 (1929).

[8] I. Bialynicki-Birula and L. Rudnicki, Statistical Complexity, edited by K. D. Sen, (Springer, New York, 2011).

[9] D. Deutsch, Phys. Rev. Lett. 50, 631 (1983).

[10] H. Maasen and J.B.M. Uffink, Phys. Rev. Lett. 60, 1103 (1988).

[11] Z. Y. Xu, W. L. Yang, and M. Feng, Phys. Rev. A 86, 012113 (2012).

[12] M. -L. Hu, and H. Fan, Phys. Rev. A 86, 032338 (2012); H. -M. Zou et al., Int. J. Theor. Phys. 53, 4302 (2014); H. -M. Zou et al., Phys. Scr. 89, 115101 (2014).

[13] G. Karpat, J. Piilo, and S. Maniscalco, EPL 111, 50006 (2015).

[14] M. Berta, M. Christandl, R. Colbeck, J. M. Renes, R. Renner, Nat. Phys. 6, 659 (2010).

[15] M. -L. Hu and H. Fan, Phys. Rev. A 87, 022314 (2013).

[16] M. -L. Hu and H. Fan, Phys. Rev. A 88, 014105 (2013).

[17] A. K. Pati, M. M. Wilde, A. R. Usha Devi, A. K. Rajagopal, and Sudha, Phys. Rev. A 86, 042105 (2012).

[18] C. Macchiavello and G. M. Palma, Phys. Rev. A 65, 050301(R) (2002).

[19] S. Daffer, K. Wodkiewicz, J. D. Cresser, J. K. McIver, Phys. Rev. A 70, 010304 (2004).

[20] C. Addis, G. Karpat, C. Macchiavello, and S. Maniscalco, Phys. Rev. A 94, 032121 (2016). 